LETTER TO JMG

\title{
Assignment of a new congenital fibrosis of extraocular muscles type 3 (CFEOM3) locus, FEOM4, based on a balanced translocation $\mathrm{t}(2 ; 13)(\mathrm{q} 37.3 ; \mathrm{q} 12.11)$ and identification of candidate genes
}

\author{
P Aubourg, M Krahn, R Bernard, K Nguyen, O Forzano, I Boccaccio, V Elague, \\ A De Sandre-Giovannoli, J Pouget, D Dépetris, M-G Mattei, N Philip, N Lévy
}

J Med Genet 2005;42:253-259. doi: 10.1136/jmg.2004.021899

\begin{abstract}
A small group of neuromuscular disorders appears to specifically target the innervation and development of the extra ocular muscles, resulting in congenital nonprogressive ophthalmoplegia and ptosis. These clinically and genetically heterogeneous disorders are classified as congenital cranial dysinnervation disorders $(\text { CCDDs })^{1}$ and include Duane's syndrome (OMIM 126800 and 604356), Moebius syndrome (OMIM 157900), congenital fibrosis of the extraocular muscles (CFEOM) (OMIM 135700, 602078, and 600638), and congenital ptosis (OMIM 300245 and 178300). The extraocular fibrosis syndromes are congenital ocular motility disorders that arise from dysfunction of the oculomotor, trochlear, and abducens nerves, and/or the muscles they innervate. Each is characterised by a specific form of restrictive paralytic ophthalmoplegia, in most cases associated with ptosis. Individuals with the classic form of congenital fibrosis of the extraocular muscles are born with bilateral ptosis and a restrictive infraductive external ophthalmoplegia, with the inability to raise either eye above the midline. In the vast majority of families, this classical form was mapped to the FEOMI locus on chromosome 12. ${ }^{2}$ Besides classical CFEOMl, variant phenotypes have been reported as CFEOM2 and CFEOM3. CFEOM2 is an autosomal recessive CFEOM, marked by a severe limitation of eye movements. ${ }^{4}$ CFEOM3 applies to patients who do not fit the typical CFEOMI or 2 criteria, owing to clinical variations (table 1). As with CFEOMI, CFEOM3 segregates as an autosomal dominant trait.

Three loci have been reported: FEOMI on chromosome 12 (CFEOM1, 2, and 3), ${ }^{6-10}$ FEOM2 on chromosome 11 (CFEOM2), ${ }^{4}$ and FEOM3 on chromosome 16 (CFEOMI and 3). ${ }^{5}{ }^{10}{ }^{11}$ CFEOM1, linked to chromosome 12, has recently been found to result from mutations in $K I F 21 A$, a member of the kinesin motor proteins superfamily. ${ }^{12}$ PHOX2A (ARIX), encoding a homeodomain transcription factor, has been identified as being involved in autosomal recessive CFEOM2, ${ }^{13}{ }^{14}$ while no gene has yet been assigned to CFEOM3, although it may be suspected that rare CFEOM3 families linked to FEOMI are caused by KIF2IA mutations (table 1). However, several families are not linked to any of the three known loci, and here we present a unique opportunity to isolate candidate genes in which interruption, misregulation, or mutations cause a novel CFEOM3 phenotype. We have investigated a family with autosomal dominant inheritance of a balanced/unbalanced translocation segregating with CFEOM through three generations. All the affected members carry a reciprocal translocation involving chromosomes $2 \mathrm{q}$ and 13q. This rearrangement is
\end{abstract}

\section{KEY POINTS}

- Congenital cranial dysinnervation disorders include congenital fibrosis of extraocular muscles, and clinically and genetically heterogeneous oculomotor disorders, mainly characterised by ophthalmoplegia and ptosis. Different phenotypes have been reported (CFEOM1-3), and three loci have been identified (FEOM1-3).

- We explored a three generation family in which members are affected with autosomal dominant CFEOM3, co-segregating with a balanced/unbalanced reciprocal translocation $\mathrm{t}(2 ; 13)$ (q37.3; $q 12.11)$.

- We hypothesised that a gene was interrupted by one of the breakpoints and caused the phenotype; both breakpoints were characterised by using a combination of molecular genetics and cytogenetics experiments.

- No gene is interrupted by the breakpoint, which lies in the well characterised 2q37 region in which a deletion causes Albright-like osteodystrophy syndrome. This makes the 2 q37 region a very unlikely locus for CFEOM3.

- We report a novel CFEOM3 locus, FEOM4, on chromosome 13, and identify several candidate genes, one of them being interrupted by the breakpoint and corresponding to a previously uncharacterised transcript of unknown function.

balanced in three affected individuals and unbalanced in one affected girl presenting with syndromic CFEOM with mental retardation and facial dysmorphism reminiscent of Albright hereditary osteodystrophy (AHO)-like syndrome ${ }^{15} 16$ (OMIM 600430). In this case, the severity of the phenotype probably reflects the loss of a small derivative chromosome (der13).

We have precisely mapped the breakpoints on both translocation partners and identified several candidate transcripts. One of these, assigned to chromosome 13, is interrupted. Meanwhile, owing to potential position effect, as previously observed in balanced translocations with abnormal phenotypes, ${ }^{17}$ other genes located apart from the breakpoints on chromosome 13 and 2 could also be involved in this CFEOM3 syndrome. 
Table 1 Clinical and genetic heterogeneity in CFEOM and classification

\begin{tabular}{lllll}
\hline CFEOM & $\begin{array}{l}\text { Mode of } \\
\text { inheritance }\end{array}$ & Main phenotype & Additional findings & Locus/gene \\
\hline CFEOM1 & $\begin{array}{l}\text { Autosomal } \\
\text { dominant }\end{array}$ & $\begin{array}{l}\text { Full penetrance. Bilateral external } \\
\text { ophtalmoplegia and ptosis } \\
\text { Infraducted position of both eyes } \\
\text { Esotropism or exotropism. Inability to } \\
\text { raise either eye above the midline. Forced } \\
\text { duction testing positive for restriction }\end{array}$ & $\begin{array}{l}\text { Excyclotropia observed } \\
\text { in one patient }\end{array}$ & FEOM1 (12q12); KIF21A \\
Bilateral ptosis. Eyes fixed primarily in \\
exotropic or outward position \\
SFEOM2
\end{tabular}

\section{SUBJECTS AND METHODS Subjects}

Four patients were clinically and genetically investigated. The pedigree is shown in fig 1 .

Patient I- 1 is the mother of II- 1 . She exhibited a congenital bilateral ptosis and partial ophthalmoplegia, with inability to raise either eye above the horizontal midline and limited lateral movements. She had undergone surgery in childhood. At 56 years of age, she was investigated in order to rule out some of the causal diagnosis of the ptosis. Creatine kinase was normal; antibodies against acetylcholine receptors were

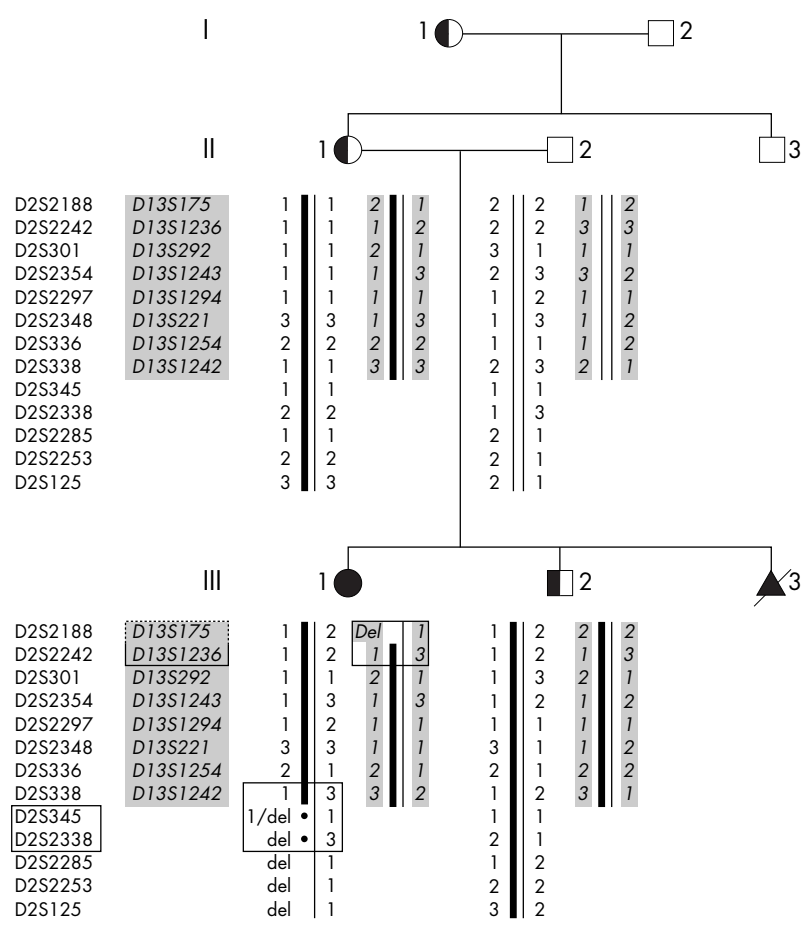

Figure 1 Pedigree of the family and segregation analysis of markers from chromosomes 2 and 13. Filled symbol: phenotype observed in III-1, including CFEOM, mental retardation and facial dysmorphism and associated to the unbalanced karyotype; half filled symbols: patients harbouring isolated CFEOM3 associated with a balanced karyotype. Marker names and haplotypes are either indicated in plain type (chromosome 2) or greyed and italics (chromosome 13). The regions containing the breakpoints are shown by squares in individual III- 1 . Subject II-3 is unaffected and the karyotype was normal. negative; and muscle biopsy did not show any sign of myopathy or mitochondriopathy. Electromyography with repetitive stimulation and further complementary study on unique fibre were normal, thus excluding the unlikely diagnosis of myasthenia.

Patient II-1 is the mother of III-1. Congenital bilateral ptosis was noted at birth. At 10 years of age, she underwent surgery for her ptosis. Ophthalmological examination (after surgery) revealed a mild ptosis and limitation of the superior rectus with a bilateral excyclotropia predominant on the right eye (fig 2a, d). CT scan of the orbits showed normal appearance of the extraocular muscles.

Patient III-l was referred to our genetic clinic soon after birth because of facial dysmorphism. She was born at 40 weeks of gestation. Oligohydramnios was noted during the third trimester. On examination, the face was asymmetric with shallow orbital ridges, arched eyebrows, exophtalmia, and ptosis. At 9 years of age, height was $127 \mathrm{~cm}$ (25th centile), weight $25 \mathrm{~kg}$ (25th centile) and HC $48.5 \mathrm{~cm}(\leqslant 2$ $\mathrm{SD}$ ). She had a thin body habitus with kyphosis and pectus excavatum. The facial appearance was unchanged, with prominent incisors and a very thin upper lip. Speech was limited to a few words. According to the mother, motor skills had regressed; she could not climb stairs without support and could hardly rise from a chair or pull herself up to stand. Neurological examination showed brisk tendon reflexes and peripheral hypertonia. Ophthalmological examination revealed a bilateral ptosis and ophthalmoplegia (fig $2 \mathrm{~b}$ ).

Patient III-2 is the second child of II- 1 . At birth, he exhibited the same facial appearance with bilateral ptosis and ophthalmoplegia. A recent ophthalmological examination describes bilateral limitation of the superior rectus and the inferior oblique, with a moderate ptosis and a bilateral excyclotropia (fig 2c, d). Mental and physical development was otherwise normal.

\section{Molecular analysis at known loci}

Because of the small size of the family, only the consistency with linkage to FEOM1 (12q12), FEOM2 (11q13.3-13.4), FEOM3 (16q24.2-24.3), PTOS1 (lp34.1-1p32) and PTOS2 (Xq24-q27.1) were tested with markers: D12S1648, D12S345, D12S59, D12S1668, D11S4139, D11S1314, DilS4184, D11S4207, D16S520, D16S476, D16S413, D16S3063, DXS1001, DXS8057, DXS1047, DXS994, DXS1192, DXS984, D1S2677, and DIS2748. Additionally, the 38 coding exons of KIF21A were PCR amplified and screened for potential variations by using denaturing high performance liquid chromatography analysis, which was 
performed on a Wave Nucleic Acid Fragment Analysis system (Transgenomic Inc) according to the manufacturer's recommendations for mutation detection. Primers used are available upon request. Additionally, exons 8, 20, and 21, containing the previously identified mutations associated to CFEOMI, ${ }^{12}$ were sequenced according to the protocol of De Sandre-Giovannoli and colleagues. ${ }^{18}$

\section{Breakpoint characterisation}

Based on the putative loss of heterozygosity in III-1, a set of markers encompassing the breakpoints on chromosomes 2 and 13 was used as previously described. All marker names are mentioned in fig 1 .

Fluorescent in situ hybridisation (FISH) using human genomic fragments cloned into bacterial artificial chromosomes (BACs) located proximal and distal to both breakpoints or encompassing them, was performed on metaphase chromosomes issued from patients lymphoblastoid cell lines, as described previously. ${ }^{19}$ Spread metaphases of the daughter carrying the unbalanced translocation were systematically compared with that of the affected mother's.

BACs were provided by the Children's hospital Oakland Research Institute Children's hospital (http://www.chori.org/ bacpac/) (see supplementary material online; Southern blots and RT-PCR procedures are also reported here.).

\section{RESULTS}

\section{Cytogenetic analysis}

A balanced translocation $\mathrm{t}(2 ; 13)(\mathrm{q} 36 ; \mathrm{q} 11)$ was observed in II-1, which was also present in I-1 and III-2. The karyotype of the affected daughter (III-1) resulted from a malsegregation of the maternal translocation and was $45 \mathrm{XX},-13,-2,+\operatorname{der}(2)$ $\mathrm{t}(2 ; 13)(\mathrm{q} 36 ; \mathrm{ql} 1)$. This rearrangement resulted in proximal 13pter-q11 and 2q36-qter monosomies. Subjects II-2 and II-3 (the brother of II-1), whose phenotypes were normal, had a normal 46,XY karyotype.

\section{Mapping exclusion to known loci}

Genotyping data were not consistent with linkage to FEOM2, FEOM 3, PTOS1, and PTOS2 loci (data not shown). As a potential linkage to the FEOMI locus could not be excluded, the full coding sequence of KIF2 $1 A^{12}$ was screened for mutations and excluded as containing the disease causing mutation. All known loci for CFEOM or isolated ptosis were then excluded either after segregation or mutation analyses.

Mapping of the breakpoints on chromosomes 2 and 13 BAC RP1 1-205L13 encompasses the breakpoint at 2q37.3

Preliminary experiments, based on the loss of heterozygosity of informative markers in III-1, indicated that the breakpoint mapped at the 2q37 region between D2S338 and D2S2338. This region covers an approximately 1.6 Mb physical interval. However, this physical distance was possibly much smaller, as D2S345 was uninformative to ascertain a loss of heterozygosity in III-1 (fig 1). Finally, FISH analysis determined that BAC RP11-205L13 encompassed the breakpoint on chromosome 2, thus definitely determining its position at $2 \mathrm{q} 37.3$ (fig 3a). At the transcriptional level, no gene that could potentially be interrupted by the breakpoint lies on RP11-205L13.

\section{BAC RP1 1-273F15 encompasses the breakpoint at $13 q 12$}

Our preliminary results indicated that the breakpoint lay in a maximum interval of $5.5 \mathrm{Mb}$ between 13cen and D13S1236, as the derl3 centromere was lost in III-1. However, as one marker (D13S175) was not sufficiently informative, it was not possible to attest definitively to its hemizygosity (fig 1). On the other hand, III-1 and III-2 have inherited the same der2 chromosome present in both III-1 and III-2 (unbalanced and balanced respectively), and we could thus hypothesise that D13S175 is deleted in III-2 rather than the maternal
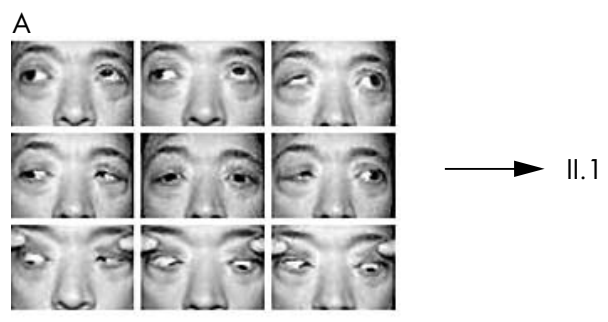

B
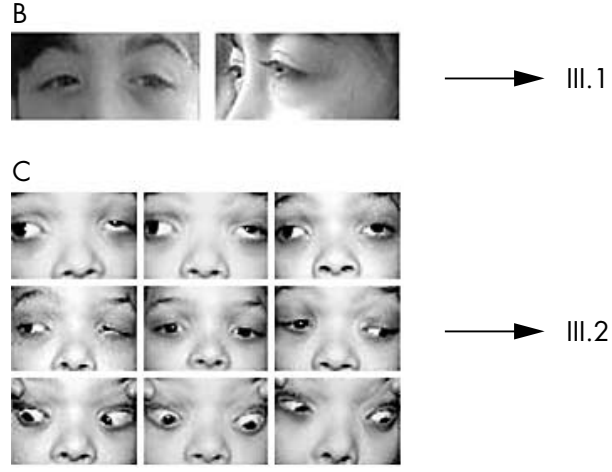

D

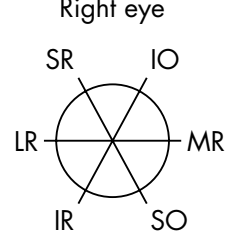

1
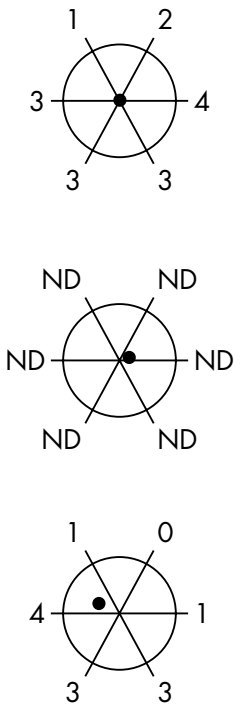
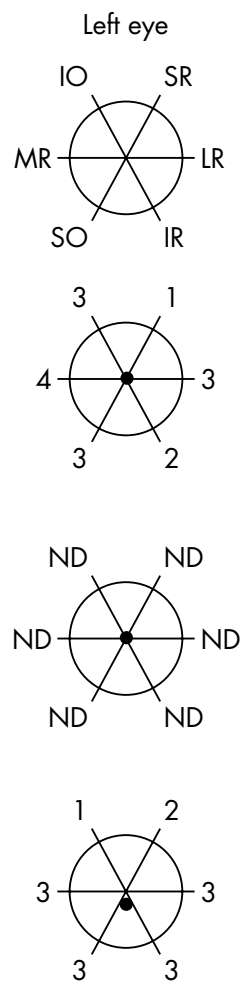

Figure 2 Clinical phenotype and diagrammatic representation of right and left eye extraocular muscle range of movement. Photographs are taken with the individual in primary gaze (centre) and attempting to focus their gaze in eight diagnostic positions (in clockwise order from top: up, up and left, left, down and left, down, down and right, right, up and right). (a) individual II-1, (c) individual III-2. Note the compensatory attitude with uplifted eyebrows. In patient III-1, arched eyebrows and shallow orbital ridges were observed, as well as exophtalmia and ptosis (b). (d) Diagrammatic representation: black dot indicates the pupillary position with the head held straight. Movements are measured from each eye's primary resting position; grade 0 (no movement) to 4 (full range of movement). IO, inferior oblique; $M R$, medial rectus; $S O$, superior oblique; $I R$, inferior rectus; $L R$, lateral rectus; $S R$, superior rectus. Reproduced with permission. 
chromosome having recombined between chromosome 13cen and D13S1236. If D13S175 is deleted, the breakpoint on chromosome 13 would lie in an approximately $2 \mathrm{Mb}$ interval between D13S175 and D13S1236.

FISH analysis with RP11-187L3 and RP11-398019 confirmed the hemizygosity at D13S175. Finally, RP11-273F15 encompassed the breakpoint on chromosome 13, exhibiting three signals on metaphase nuclei from II.l, respectively corresponding to the normal chromosome 13 and both derivative chromosomes (fig $3 \mathrm{~b}$ ). The maximum size of the critical interval was thus reduced to $65 \mathrm{~kb}$ between the proximal end of the BAC RP11-273F15 and D13S1236. FISH experiments are summarised in fig 3(c).

Southern blot analyses with overlapping probes selected to span the full critical breakpoint interval allowed identification of junction fragments from chromosomes 13 and 2 (fig 3d), both of which were subsequently sequenced and annotated by comparing from sequence databases after BLAST analysis (http://ncbi.nlm.nih.gov/BLAST/).

Candidate gene analysis at or next to the breakpoints At the transcriptional level, no gene was interrupted by the breakpoint on chromosome 2 . The most proximal genes are located $100 \mathrm{~kb}$ upstream and $400 \mathrm{~kb}$ downstream of the breakpoint. They respectively encode a chemokine orphan receptor I (CMKORI; Unigene accession no. Hs.231853), a putative protein member of the $G$ protein coupled receptor family, and a COP9 constitutive photomorphogenic homologue subunit 8 (COPS8; Unigene accession no. Hs.445109), one of the eight subunits of the COP9 signalosome, a highly conserved protein complex that functions as an important regulator in multiple signalling pathways.

On chromosome 13, FGF9 (OMIM 600921; Unigene accession no. Hs.111), a member of the fibroblast growth factor family, maps $390 \mathrm{~kb}$ upstream of the breakpoint. On the other side, SGCG, encoding $\gamma$-sarcoglycan (UniGene accession no. Hs.37167) is located $1 \mathrm{Mb}$ downstream. A physical, genetic, and transcriptional map of the chromosome 13 interval is shown in fig 4, according to the UCSC Human Genome Project Working Draft (http://genome.ucsc.edu/).

Although no known gene was interrupted by the breakpoint at 13q12.11, one uncharacterised cDNA (AK054845) of unknown function is interrupted by the breakpoint. AK054845 shares one homologous exon with BC035104, another uncharacterised cDNA that is not interrupted by the breakpoint. We sequenced both mRNAs and their intron - exon boundaries and identified consensus donor (AG) and acceptor (GT) splicing sites. This allowed elimination of a potential genomic sequence that could have been annotated as an mRNA in databases. AK054845 and BC035104 are alternatively spliced mRNAs from the same gene as validated after RT-PCR experiments using a combination of primers (fig 4d), and are expressed in various tissues including brain, skeletal muscle, and heart (data not shown). Intriguingly, no putative function has been assigned to these transcripts, nor could it be easily predicted. In particular, no clear open reading frame or homology to a known gene family could be identified.

\section{DISCUSSION}

Contrary to apparently balanced de novo translocations associated with abnormal phenotypes, inherited balanced translocations are a strong argument to indicate that a gene, interrupted by one of the breakpoints or located next to the breakpoints (position effect), is disease causing. Indeed, a possibility exists that de novo translocations are randomly associated with abnormal phenotypes.

In the present study, the autosomal dominant mode of inheritance of the $t(2 ; 13)$ translocation associated with CFEOM3 thus represents a powerful tool to identify the
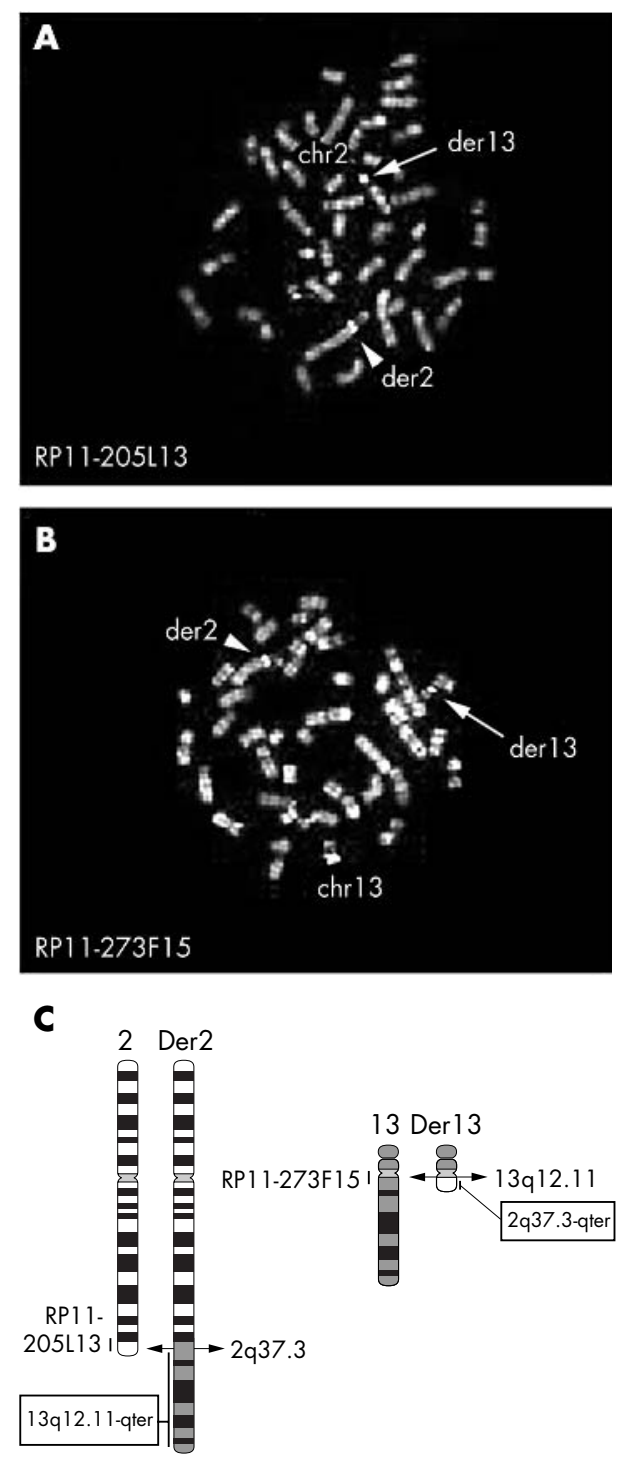

D

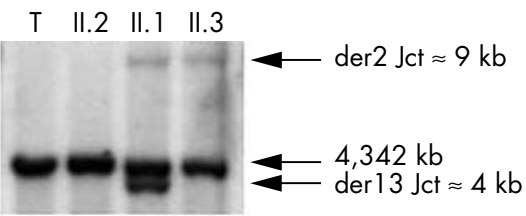

Figure 3 FISH analysis with BACs from chromosome 2 and 13. (a) BAC RP1 1-205L13 contains a human sequence from chromosome 2, encompassing the breakpoint. Three signals are observed on metaphases from individual II-1 on normal chromosome 2, der2 (arrowhead), and der13 (arrow) respectively. (b) BAC RP1 1-273F15 contains a human sequence from chromosome 13, encompassing the breakpoint. Three signals are observed on metaphase chromosomes from individual II-1 on normal chromosome 13, der13 (arrow), and der2 arrowhead), respectively. (c) Schematic representation of the chromosomal translocation $(2 ; 13)$ and breakpoint mapping.

(d) Southern blot showing the junction fragments from the breakpoints. Both junction fragments are present from the individual II-1, carrying both der 2 and der 13 chromosomes in the balanced translocation (lane 3) while individual III-1 harbours only the junction fragment corresponding to the der2 because she lost the der13 in her unbalanced karyotype. 
disease gene. The pedigree is consistent with both autosomal and X linked dominant inheritance; however, as the phenotypic trait segregated with a balanced $\mathrm{t}(2 ; 13)$ translocation, a disruption of a gene located at or next to (position effect) one of the breakpoints was more likely to be involved as the cause of CFEOM in this family.

Hereditary forms of have been reported, and both autosomal dominant and $\mathrm{X}$ linked dominant inheritance of congenital bilateral isolated ptosis have been described. The $\mathrm{X}$ linked form is assigned to the Xq24-q27.1 region, ${ }^{20}$ whereas autosomal dominant ptosis is assigned to the 1p32-p34.2 region. ${ }^{21}$ In the family reported here, all affected patients exhibit non-progressive restrictive ophthalmoplegia and ptosis, primarily affecting muscles in the oculomotor distribution. This clinical picture fits the definition of CFEOM. ${ }^{9}$ Three different phenotypes (CFEOMl-3) have been delineated. Although most of the families with the same phenotype usually map to distinct loci, genetic heterogeneity among families clinically diagnosed as CFEOMI and CFEOM3 was recently demonstrated (clinical and genetic heterogeneity in CFEOM is summarised in table 1). CFEOM3 thus maps to FEOM1, FEOM3, and now FEOM4. Recently, mutations in KIF21A have been reported in CFEOMl linked to FEOM1. ${ }^{12}$ KIF21A encodes a member of the kinesin superfamily, which are major axonal transport proteins. CFEOM2, which is a recessively inherited atypical and severe phenotype with eyes fixed in exotropia, is caused by mutations in the ARIX gene (PHOX2A) on $11 \mathrm{q} 13 .^{13}$

The family reported here fulfils the diagnostic criteria for CFEOM, and some members are very similar to some of the members of the CFEOM3 families that map to both the FEOMI and FEOM3 loci. These findings are consistent with the existence of at least a fourth locus for CFEOM, and a third locus for CFEOM3. The phenotype of patient III-1 provides some clues to the identification of the precise locus. Indeed, this girl has a distal 2q monosomy and a proximal 13q monosomy. Some clinical manifestations, namely seizures, developmental delay, and hypotonia, with some dysmorphic features such as thin upper lip and syndactyly, belong to the well delineated AHO-like syndrome, caused by 2qter deletions. ${ }^{15}{ }^{16}$ As ptosis is not associated with 2qter deletions, it is, presumably, the consequence of a loss of heterozygosity at chromosome 13q, making the 13q breakpoint a better candidate for the gene locus. Moreover, no gene was found to be interrupted by the breakpoint on chromosome 2. However, a position effect may be inferred, involving the genes adjacent to the breakpoint. In this case, this mechanism might concern CMKORl, located $100 \mathrm{~kb}$ centromeric to the breakpoint, and COPS8, located $400 \mathrm{~kb}$ telomeric from the breakpoint on chromosome 2. CMKORI is a member of the $G$ protein coupled receptor family and is considered an orphan receptor and a co-receptor for HIV. ${ }^{22}$ Translocations involving this gene and HMGA2 on chromosome 12 have been observed in lipomas. ${ }^{23}$

COPS8 is one of the subunits of COP9 signalosome, an important regulator in multiple signalling pathways. ${ }^{24}$ The structure and function of COP9 signalosome is similar to that of the 19S regulatory particle of the $26 \mathrm{~S}$ proteasome. Because of their predicted or known function, plus the fact that these genes are deleted in AHO-like affected patients without CFEOM, they are unlikely to be involved in the pathogenesis in this family.

On chromosome 13, SGCG maps $1 \mathrm{Mb}$ telomeric from the breakpoint and encodes $\gamma$-sarcoglycan, a dystrophin associated glycoprotein of which a homozygous defect leads to early onset limb girdle muscular dystrophy type $2 \mathrm{C}^{25}$ (OMIM 253700). This condition is characterised by an autosomal recessive mode of inheritance and neither ptosis nor ophthalmoplegia have ever been found as part of this phenotype. In addition, our affected patients do not present with any muscular dystrophy or limb girdle muscle weakness, and creatine kinase results were in the normal range. Taken together, these facts make $S G C G$ a very unlikely candidate gene for FEOM4.

FGF9 maps $390 \mathrm{~kb}$ centromeric from the breakpoint, and encodes a member of the fibroblast growth factor family. It has a growth stimulating effect on cultured glial cells and, in the nervous system, is produced mainly by neurones and might be important for glial cell development. ${ }^{26}{ }^{27}$ It is an attractive candidate for CFEOM as being a disorder of cranial

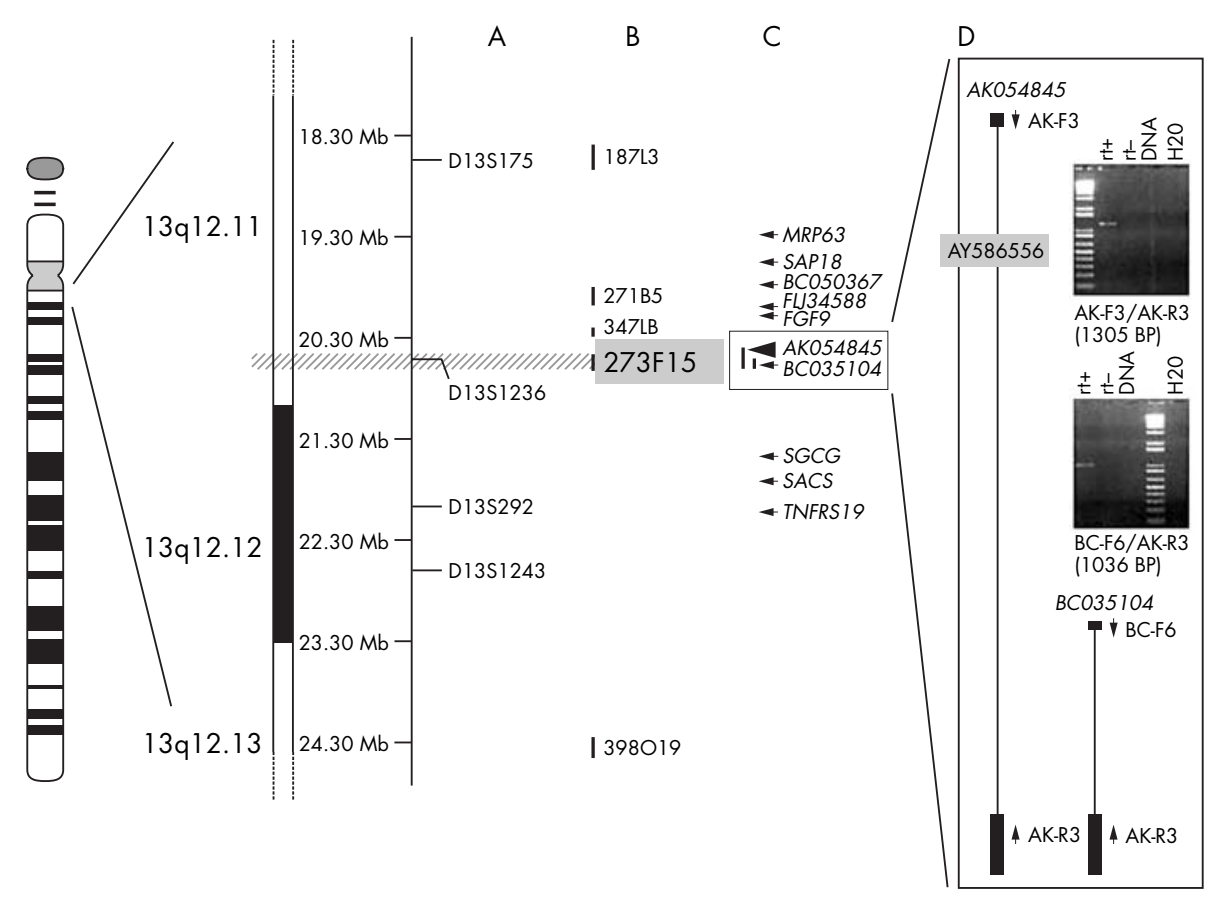

Figure 4 Integrated genetic, physical, and transcriptional map surrounding the breakpoint translocation on chromosome 13. (a) Genetic map: genetic markers are placed on the bar corresponding to the physical distance according to the Human Genome Working Draft (http://genome.ucsc. edu/). (b) The position of the BACs is indicated with respect to the genetic markers. Only the BACs used in FISH analysis are mentioned. Diagonal hatched bar: minimal interval covering the breakpoint (BAC RP1 1-273F15).

(c) Electronic mapping of most proximal or distal genes and transcripts. Genbank accession numbers are mentioned for uncharacterised transcripts. (d) Reverse transcriptase PCR experiments to ascertain the transcript status for AK054845 and BC035104. The primer sets are indicated according to the schematic representation of both transcripts. The exact sequence containing the breakpoint on chromosome 13 is identified as AY586556 in Genbank (http://www.ncbi.nlm.nih.gov/ Genbank/) and positioned at the corresponding position within AK054845. 
nerve development, although FGF9 knockout mice harbour a male to female sex reversal phenotype, suggesting a major role in testicular embryogenesis. ${ }^{28}$

Interestingly, one transcript of unknown function, AKO54845, is interrupted by the translocation breakpoint. This transcript does not encode any known protein, nor can any putative open reading frame be predicted. AKO54845 is a two block transcript containing consensus acceptor and donor splicing sequences and shares $2 \mathrm{~kb}$ homology (the full last exon) with BC035104 in humans. Indeed, both cDNAs correspond to alternative transcripts from a same gene. Despite absence of homology to known proteins or genes from other species, AK054845 might be involved in the pathogenesis of CFEOM3. Expression studies by reverse transcriptase PCR indicate that it is expressed at low levels in brain, skeletal muscle, and heart. Additionally, it is well documented that non-coding RNAs may be functionally active as splicing enhancers, transcription activators, or inhibitors or neuronal differentiation. ${ }^{29}{ }^{30}$ Despite these hypotheses, there is no clear evidence yet that such a mechanism could be related to AK054845, thus making unclear the functional impact related to the heterozygous disruption of this mRNA.

In affected patients from our family, the extraocular muscles appeared normal on computed tomography scan in one patient (II-1), with no sign of fibrosis, reinforcing the hypothesis of developmental defect of the oculomotor nuclei rather than a disorder of the extraocular muscles.

In summary, we report a new candidate locus for CFEOM. Indeed, the locus on chromosome $13 \mathrm{q} 12$ is likely to be involved in the pathogenesis of CFEOM3, as our study demonstrated that the breakpoint on chromosome 2 lies within the AHO-like syndrome critical interval, in which ptosis does not belong to the clinical synopsis.

Identifying the genes involved in CFEOM provides new hints towards the understanding of the CCDDs. The precise identification of the CFEOM3 genes should help to elucidate the pathophysiology of this disorder and clarify the basis of clinical heterogeneity observed in CFEOM. In addition, the ability to test the genetic segregation at the FEOM4 locus in families previously shown to be unlinked to any of the three FEOM loci will allow better and more precise genetic counselling. Further studies should focus on mutation screening of AK054845, to pinpoint this transcript as possibly containing disease causing mutations or excluding such a hypothesis.

\section{ACKNOWLEDGEMENTS}

The authors are extremely grateful to patients for their invaluable cooperation. We are indebted to E C Engle for critical reading of the manuscript and helpful comments. We warmly acknowledge P Negre and C Thouveny, and the cell and DNA core laboratory of MarseilleTimone for preparing and providing us with DNAs and cell lines. This study was supported in part by the Association Française contre les Myopathies (grant no. 9183), the Institut National de la Sante et de la Recherche Médicale, and the Assistance Publique des Hopitaux de Marseille. A DeSandre-Giovannoli has received a fellowship grant from the Association Française contre les Myopathies.

\footnotetext{
Authors' affiliations

P Aubourg, M Krahn, R Bernard, I Boccaccio, V Elague, A De SandreGiovannoli, D Dépetris, M-G Mattei, N Philip, N Lévy, Inserm U491:

"Genetique Medicale et Developpement", Faculte de Medecine de Marseille, IFR 125, 13385 Marseille cedex 05, France

M Krahn, R Bernard, K Nguyen, N Philip, N Lévy, Departement de Genetique Medicale, Hopital d'Enfants de la Timone, IFR 125, 13385 Marseille cedex 05, France

O Forzano, Service d'Ophtalmologie, Hopital de la Timone, Marseille, France
}

J Pouget, Service des Maladies Neuromusculaires, Hopital de la Timone, Marseille, France

Competing interests: none declared

Correspondence to: Dr N Lévy, INSERM U491 "Genetique Medicale et Developpement", Faculté de Médecine de Marseille, 13385 Marseille cedex 05, France; nicolas.levy@medecine.univ-mrs.fr

Received 21 April 2004

Accepted for publication 20 May 2004

\section{REFERENCES}

1 Gutowski NJ, Bosley TM, Engle EC. 110th ENMC International Workshop: the Congenital Cranial Dysinnervation Disorders (CCDDs), Naarden, The Netherlands, 25-27 October, 2002. Neuromuscul Disord 2003;13:573-8.

2 Engle EC, Kunkel LM, Specht LA, Beggs AH. Mapping a gene for congenital fibrosis of the extraocular muscles to the centromeric region of chromosome 12. Nat Genet 1994:7:69-73.

3 Engle EC, Marondel I, Houtman WA, de Vries B, Loewenstein A, Lazar M, Ward DC, Kucherlapati R, Beggs AH. Congenital fibrosis of the extraocular muscles (autosomal dominant congenital external ophthalmoplegia): genetic homogeneity, linkage refinement, and physical mapping on chromosome 12 Am J Hum Genet 1995;57:1086-94.

4 Wang SM, Zwaan J, Mullaney PB, Jabak MH, Al-Awad A, Beggs AH, Engle EC Congenital fibrosis of the extraocular muscles type 2, an inherited exotropic strabismus fixus, maps to distal 11q13. Am J Hum Genet 1998;63:517-25.

5 Doherty EJ, Macy ME, Wang SM, Dykeman CP, Melanson MT, Engle EC. CFEOM3: a new extraocular congenital fibrosis syndrome that maps to 16q24.2-q24.3. Invest Ophthalmol Vis Sci 1999;40:1687-94.

6 Venkatesh CP, Pillai VS, Raghunath A, Prakash VS, Vathsala R, PericakVance MA, Kumar A. Clinical phenotype and linkage analysis of the congenital fibrosis of the extraocular muscles in an Indian family. Mol Vis 2002;8:294-7.

7 Traboulsi El, Lee BA, Mousawi A, Khamis AR, Engle EC. Evidence of genetic heterogeneity in autosomal recessive congenital fibrosis of the extraocular muscles. Am J Ophthalmol 2000;129:658-62.

8 Sener EC, Lee BA, Turgut B, Akarsu AN, Engle EC. A clinically variant fibrosis syndrome in a Turkish family maps to the CFEOM1 locus on chromosome 12 Arch Ophthalmol 2000;118:1090-7.

9 Engle EC. Applications of molecular genetics to the understanding of congenital ocular motility disorders. Ann N Y Acad Sci 2002;956:55-63.

10 Engle EC, Mclntosh N, Yamada K, Lee BA, Johnson R, O'Keefe M, Letson R, London A, Ballard E, Ruttum M, Matsumoto N, Saito N, Collins ML, Morris L, Del Monte M, Magli A, de Berardinis T. CFEOM1, the classic familial form of congenital fibrosis of the extraocular muscles, is genetically heterogeneous but does not result from mutations in ARIX. BMC Genet 2002;3:3.

11 Mackey DA, Chan WM, Chan C, Gillies WE, Brooks AM, O'Day J, Engle EC Congenital fibrosis of the vertically acting extraocular muscles maps to the FEOM3 locus. Hum Genet 2002;1 10:510-12.

12 Yamada K, Andrews C, Chan WM, McKeown CA, Magli A, de Berardinis T, Loewenstein A, Lazar $M, O^{\prime}$ Keefe $M$, Letson $R$, London A, Ruttum $M$, Matsumoto N, Saito N, Morris L, Del Monte M, Johnson RH, Uyama E Houtman WA, de Vries B, Carlow TJ, Hart BL, Krawiecki N, Shoffner J, Vogel MC, Katowitz J, Goldstein SM, Levin AV, Sener EC, Ozturk BT, Akarsu AN, Brodsky MC, Hanisch F, Cruse RP, Zubcov AA, Robb RM, Roggenkaemper P, Gottlob I, Kowal L, Battu R, Traboulsi El, Franceschini P, Newlin A, Demer JL, Engle EC. Heterozygous mutations of the kinesin KIF21A in congenital fibrosis of the extraocular muscles type 1 (CFEOM1). Nat Genet 2003;35:318-21.

13 Nakano M, Yamada K, Fain J, et al. Homozygous mutations in ARIX(PHOX2A) result in congenital fibrosis of the extraocular muscles type 2. Nat Genet $2001 ; 29: 315-20$.

14 Yazdani A, Chung DC, Abbaszadegan MR, Al-Khayer K, Chan WM, Yazdani M, Ghodsi K, Engle EC, Traboulsi El. A novel PHOX2A/ARIX mutation in an Iranian family with congenital fibrosis of extraocular muscles type 2 (CFEOM2). Am J Ophthalmol 2003;136:861-5.

15 Wilson LC, Leverton K, Oude Luttikhuis ME, Oley CA, Flint J, Wolstenholme J, Duckett DP, Barrow MA, Leonard JV, Read AP, Trembath RC. Brachydactyly and mental retardation: an Albright hereditary osteodystrophy-like syndrome localized to 2q37. Am J Hum Genet 1995;56:400-7.

16 Phelan MC, Rogers RC, Clarkson KB, Bowyer FP, Levine MA, Estabrooks LL, Severson MC, Dobyns WB. Albright hereditary osteodystrophy and del(2) (q37.3) in four unrelated individuals. Am J Med Genet 1995;58:1-7.

17 Kleinjan DJ, van Heyningen V. Position effect in human genetic disease. Hum Mol Genet 1998:7:1611-18.

18 De Sandre-Giovannoli A, Chaouch M, Kozlov S, Vallat JM, Tazir M, Kassouri N, Szepetowski P, Hammadouche T, Vandenberghe A, Stewart CL, Grid D, Levy N. Homozygous defects in LMNA, encoding lamin A/C nuclearenvelope proteins, cause autosomal recessive axonal neuropathy in human (Charcot-Marie-Tooth disorder type 2) and mouse. Am J Hum Genet 2002;70:726-36.

19 Mignon-Ravix C, Mugneret F, Stavropoulou C, Depetris D, Khau Van Kien P Mattei MG. Maternally inherited duplication of the possible imprinted $14 \mathrm{q} 31$ region. J Med Genet 2001;38:343-7. 
20 McMullan TF, Collins AR, Tyers AG, Robinson DO. A novel X-linked dominant condition: X-linked congenital isolated ptosis. Am J Hum Genet 2000;66: 1455-60.

21 Engle EC, Castro AE, Macy ME, Knoll JH, Beggs AH. A gene for isolated congenital ptosis maps to a 3-cM region within 1p32-p34.1. Am J Hum Genet 1997;60:1150-7.

22 Shimizu N, Soda Y, Kanbe K, Liu HY, Mukai R, Kitamura T, Hoshino H. A putative $G$ protein-coupled receptor, $R D C l$, is a novel coreceptor for human and simian immunodeficiency viruses. J Virol 2000;74:619-26.

23 Broberg K, Zhang M, Strombeck B, Isaksson M, Nilsson M, Mertens F, Mandahl N, Panagopoulos I. Fusion of RDC1 with HMGA2 in lipomas as the result of chromosome aberrations involving 2q35-37 and 12q13-15. Int J Oncol 2002;21:321-6.

24 Gizatullin RZ, Muravenko OV, Al-Amin AN, Wang F, Protopopov Al, Kashuba VI, Zelenin AV, Zabarovsk ER. Human COP' subunit 8 homolog gene SGN8 Map position 2q37. Chromosome Res 2000;8:559.
25 Noguchi S, McNally EM, Ben Othmane K, Hagiwara Y, Mizuno Y, Yoshida M, Yamamoto H, Bonnemann CG, Gussoni E, Denton PH, Kyriakides T, Middleton L, Hentati F, Ben Hamida M, Nonaka I, Vance JM, Kunkél LM, Ozawa E. Mutations in the dystrophin-associated protein gammasarcoglycan in chromosome 13 muscular dystrophy. Science 1995;270:819-22.

26 Nakamura S, Todo T, Motoi Y, Haga S, Aizawa T, Ueki A, Ikeda K Glial expression of fibroblast growth factor-9 in rat central nervous system. Glia 1999;28:53-65.

27 Du Y, Dreyfus CF. Oligodendrocytes as providers of growth factors. J Neurosci Res 2002;68:647-54

28 Colvin JS, Green RP, Schmahl J, Capel B, Ornitz DM. Male-to-female sex reversal in mice lacking fibroblast growth factor 9. Cell 2001;104:875-89.

29 Eddy SR. Non-coding RNA genes and the modern RNA world. Nat Rev Genet 2001;2:919-29.

30 Kuwabara T, Hsieh J, Nakashima K, Taira K, Gage FH. A small modulatory dsRNA specifies the fate of adult neural stem cells. Cell 2004;1 16:779-93. 